\title{
Hepatic stellate cells promote upregulation of epithelial cell adhesion molecule and epithelial-mesenchymal transition in hepatic cancer cells
}

\author{
TERUYA NAGAHARA, HIDENORI SHIRAHA, HIROAKI SAWAHARA, DAISUKE UCHIDA, YASUTO TAKEUCHI, \\ MASAYA IWAMURO, JUNRO KATAOKA, SHIGERU HORIGUCHI, TAKESHI KUWAKI, HIDEKI ONISHI, \\ SHINICHIRO NAKAMURA, AKINOBU TAKAKI, KAZUHIRO NOUSO and KAZUHIDE YAMAMOTO
}

Department of Gastroenterology and Hepatology, Okayama University Graduate School of Medicine, Shikata-cho, Kita-ku, Okayama 700-8558, Japan

Received May 12, 2015; Accepted June 26, 2015

DOI: $10.3892 / o r .2015 .4126$

\begin{abstract}
Microenvironment plays an important role in epithelial-mesenchymal transition (EMT) and stemness of cells in hepatocellular carcinoma (HCC). Epithelial cell adhesion molecule (EpCAM) is known as a tumor stemness marker of HCC. To investigate the relationship between microenvironment and stemness, we performed an in vitro co-culture assay. Four HCC cell lines (HepG2, Hep3B, HuH-7 and PLC/ PRF/5) were co-cultured with the TWNT-1 immortalized hepatic stellate cells (HSCs), which create a microenvironment with HCC. Cell proliferation ability was analyzed by flow cytometry (FCM) and 3-(4,5-dimethylthiazol-2-yl)-2,5-diphenyltetrazolium bromide (MTT) assay, while migration ability was assessed by a wound healing assay. Expression of EpCAM was analyzed by immunoblotting and FCM. HCC cell lines were co-cultured with TWNT-1 treated with small interfering RNA (siRNA) for TGF- $\beta$ and HB-EGF; we then analyzed proliferation, migration ability and protein expression using the methods described above. Proliferation ability was unchanged in HCC cell lines co-cultured with TWNT-1. Migration ability was increased in HCC cell lines (HepG2, Hep3B, HuH-7 and PLC/PRF/5) directly $(216.2 \pm 67.0,61.0 \pm 22.0,124.0 \pm 66.2$ and $51.5 \pm 40.3 \%)$ and indirectly $(102.5 \pm 22.0,84.6 \pm 30.9,86.1 \pm 25.7$ and $73.9 \pm 29.7 \%$ ) co-cultured with TWNT-1 compared with the HCC uni-culture. Immunoblot analysis revealed increased EpCAM expression in the HCC cell lines co-cultured with TWNT-1. Flow cytometry revealed that the population of E-cadherin $/ \mathrm{N}$-cadherin ${ }^{+}$and EpCAM-positive cells increased and accordingly, EMT and stemness in the HCC cell line
\end{abstract}

Correspondence to: Dr Hidenori Shiraha, Department of Gastroenterology and Hepatology, Okayama University, 2-5-1 Shikata-cho, Kita-ku, Okayama 700-8558, Japan

E-mail: hshiraha@md.okayama-u.ac.jp

Key words: hepatocellular carcinoma, hepatic stellate cell, niche, Notch, EpCAM, cancer stem cells were activated. These results were similar in the directly and indirectly co-cultured samples, indicating that humoral factors were at play. Conversely, HCC cell lines co-cultured with siRNA-treated TWNT-1 showed decreased migration ability, a decreased population of EpCAM-positive and E-cadherin $/$ $\mathrm{N}$-cadherin ${ }^{+}$cells. Taken together, humoral factors secreted from TWNT-1 promote upregulation of EpCAM and EMT in hepatic cancer cells.

\section{Introduction}

Hepatocellular carcinoma (HCC) is the third most frequent cause of cancer-related death worldwide $(1,2)$. There are several treatment modalities for HCC, including surgical resection, radiofrequency ablation, transcatheter arterial chemoembolization and percutaneous ethanol injection therapy. Recent advances in these treatment modalities have improved the prognosis of HCC, yet prognosis for advanced HCC remains poor (1). Several studies investigated the factors associated with refractory HCC have shown that CD133 and epithelial cell adhesion molecule (EpCAM) are related to the poor prognosis (3-5).

Stemness is an essential characteristic of stem cells to renew themselves and differentiate with multipotency. EpCAM is a cell surface marker expressed on human hepatic stem cells and is a known marker to identify cancer stem cells (CSCs) in HCC (6). EpCAM is related to tumorigenesis and metastasis, and its expression is a prognostic factor for HCC (3-5). EpCAM is a downstream effector of the Notch signaling cascade. Activation of Notch signaling enhances cellular stemness and epithelial-mesenchymal transition (EMT) by inducing EpCAM expression in both pancreatic cancer (7) and HCC (8). Moreover, other investigators have reported that EpCAM plays an important role in $\operatorname{EMT}(9,10)$.

Cancer progression is related to genetic changes within cancer cells as well as in the microenvironment. Stromal cells in the cancer microenvironment facilitate the development of cancer EMT (11). Cancer cells that undergo EMT also acquire a stem cell-like phenotype (12), suggesting that cancer cell-microenvironment interactions are important in cancer 
progression. In various types of cancers, such as intrahepatic cholangiocarcinoma (13), oligodendroglioma (14), pancreatic cancer (15), in vitro co-culture assays of cancer cell lines and cells in the cancer microenvironment increases EMT. In the present study, we hypothesized that the microenvironment associated with HCC enhances EMT. Hepatic stellate cells (HSCs) are liver-specific mesenchymal cells located in perisinusoidal and portal areas. HSCs play an important role in the stem cell niche for hepatic progenitor cells and hepatocytes. In addition, HSCs are known to present histopathologically among HCC tissue (16), and are thought to make a niche for hepatic cancer cells. Therefore, in the present study, we investigated the interaction between HSCs and HCC cells.

\section{Materials and methods}

Cell lines and culture. The human HCC cell lines HepG2, Hep3B, HuH-7 and PLC/PRF/5 were obtained from American Type Culture Collection (ATCC; Manassas, VA, USA). Immortalized human HSC cells (TWNT-1) were a generous gift from Dr Naoya Kobayashi from the Department of Gastroenterological Surgery, Okayama University School of Medicine. Cells were maintained in high glucose Dulbecco's modified Eagle's medium (DMEM; Invitrogen, Carlsbad, CA, USA) supplemented with $10 \%$ heat-inactivated fetal bovine serum (FBS), 1\% non-essential amino acids, penicillin/ streptomycin solution (both from Sigma-Aldrich, St. Louis, MO, USA). Cells were cultured at $37^{\circ} \mathrm{C}$ in an atmosphere of $5 \% \mathrm{CO}_{2}$ and $95 \%$ air. The cells were treated under restricted serum conditions with $0.5 \%$ dialyzed FBS for $24 \mathrm{~h}$ before the experiment when necessary.

Direct co-culture of hepatic cancer cells and HSCs. HCC cell lines [400,000 cells/well (HepG2), 200,000 cells/well (Hep3B, HuH-7 and PLC/PRF/5)] and TWNT-1 (50,000 cells/well) were seeded in 6-well culture plates (353046; Corning, Corning, NY, USA) in DMEM supplemented with $0.5 \%$ dialyzed FBS and 1\% supplements as previously described, and incubated for 3 days. If required, HSCs were pre-treated with mitomycin $\mathrm{C}$ before they were used for assays in order to inhibit self-proliferation. After this, cells were seeded and cultured in this manner in case of direct co-culture unless otherwise specified.

Indirect co-culture of hepatic cancer cells and HSCs. HCC cell lines [400,000 cells/well (HepG2), 200,000 cells/well (Hep3B, HuH-7 and PLC/PRF/5)] were seeded in 6-well culture plates in DMEM supplemented with $0.5 \%$ dialyzed FBS and $1 \%$ supplements as previously described. TWNT-1 $\left(50,000\right.$ cells/well) were seeded into the Cell Culture Insert ${ }^{\mathrm{TM}}$ of $1.0-\mu \mathrm{m}$ pore size (353102; Corning) in the same medium as used for HCC cells, inserted into the 6 -well plates where HCC cells were seeded, and incubated for 3 days. After this, cells were seeded and cultured in this manner in case of indirect co-culture unless otherwise specified.

Immunoblot analysis. HCC cells were seeded in 6-well culture plates and uni-cultured and indirectly co-cultured with TWNT-1, and grown to confluence. The HCC cells were washed twice with cold Dulbecco's phosphate-buffered saline
(DPBS) and lysed in $100 \mu \mathrm{l}$ of sample buffer [100 mM Tris- $\mathrm{HCl}$ (pH 6.8), 10\% glycerol, 4\% sodium dodecyl sulfate (SDS), $1 \%$ bromophenol blue and $10 \% \beta$-mercaptoethanol]. The samples were resolved by SDS-polyacrylamide gel electrophoresis and transferred to a polyvinylidene difluoride (PVDF) membrane (Bio-Rad, Hercules, CA, USA). The membranes were blocked using PVDF blocking reagent Can Get Signal ${ }^{\mathrm{TM}}$ (Toyobo, Osaka, Japan) for $1 \mathrm{~h}$. The membranes were then incubated with antibodies against cleaved Notch [\#2421; Cell Signaling Technology (CST), Danvers, MA, USA], Hes1 (ab49170; Abcam, Cambridge, UK), Twist1 [sc-6070; Santa Cruz Biotechnology (SCBT), Dallas, TX, USA], E-cadherin (\#3195), N-cadherin (\#13116), EpCAM (\#2929) and $\beta$-actin (\#4967S) (all from CST) for $1 \mathrm{~h}$ at room temperature. The membranes were washed 3 times with TBS-T and probed with horseradish peroxidase-conjugated secondary antibody (\#7074S; CST) before being developed with an ECL blotting detection system (Amersham Biosciences, Piscataway, NJ, USA) using enhanced chemiluminescence.

Gene silencing of TGF- $\beta$ and HB-EGF with small interfering RNA (siRNA). TWNT-1 was transfected with scrambled negative control siRNA and TGF- $\beta 1 / 2 / 3$, and HB-EGF siRNA, respectively (sc-37007, sc-44146 and sc-39420; SCBT). siRNAs were transfected into cells using a siRNA transfection reagent and siRNA transfection medium (sc-45064; SCBT). Cells were seeded at 200,000 cells/well in 6-well culture plates and incubated for $24 \mathrm{~h}$ before the $5 \mathrm{~h}$ transfection with scrambled negative control, TGF- $\beta 1 / 2 / 3$ and HB-EGF siRNA, and then used for assays after additional incubation for $48 \mathrm{~h}$.

MTT assay. Cell proliferation ability was evaluated with the 3-(4,5-dimethylthiazol-2-yl)-2,5-diphenyltetrazolium bromide (MTT) assay. HCC cell lines were seeded at 40,000 cells/well in $500 \mu$ l of DMEM supplemented with $1 \%$ dialyzed FBS in 24-well culture plates (353047; Becton-Dickinson, Franklin Lakes, NJ, USA). TWNT-1 (30,000 cells/well) were seeded into the Cell Culture Insert ${ }^{\mathrm{TM}}$ of $1.0-\mu \mathrm{m}$ pore size (353104; Corning) in the same medium as used for HCC cells, and plated into the 24-well plates where the HCC cells were seeded. After $48 \mathrm{~h}$ of quiescence, $50 \mu \mathrm{l}$ of MTT $(5 \mathrm{mg} / \mathrm{ml}$ in PBS) was added to each well, and the wells were incubated for an additional $3.5 \mathrm{~h}$ at $37^{\circ} \mathrm{C}$. The purple-blue MTT formazan precipitate was dissolved in $500 \mu \mathrm{l}$ of dimethylsulfoxide (Sigma-Aldrich) and applied in $100 \mu 1$ volumes in a 96-well culture plate in the fourth replicate. The activity of mitochondria, used as a measure of cellular growth and viability, was evaluated by measuring optical density at $570 \mathrm{~nm}$ with a microplate reader (Model 680 microplate reader; Bio-Rad).

Migration assay. Cell migration ability was evaluated by an in vitro wound healing assay. HCC cell lines (HepG2, Hep3B, HuH-7 and PLC/PRF/5) were seeded at 500,000, 600,000, 200,000 and 600,000 cells/well, respectively, in 6-well culture plates then uni-cultured, directly and indirectly co-cultured with TWNT-1 (50,000/well) in DMEM supplemented with $10 \%$ FBS. TWNT-1 was pre-treated with mitomycin C before use in the direct co-culture assays to inhibit self-proliferation. After cells grew to confluence, the cell monolayer was mechanically scratched with a sterile $200 \mu \mathrm{l}$ pipette tip and 

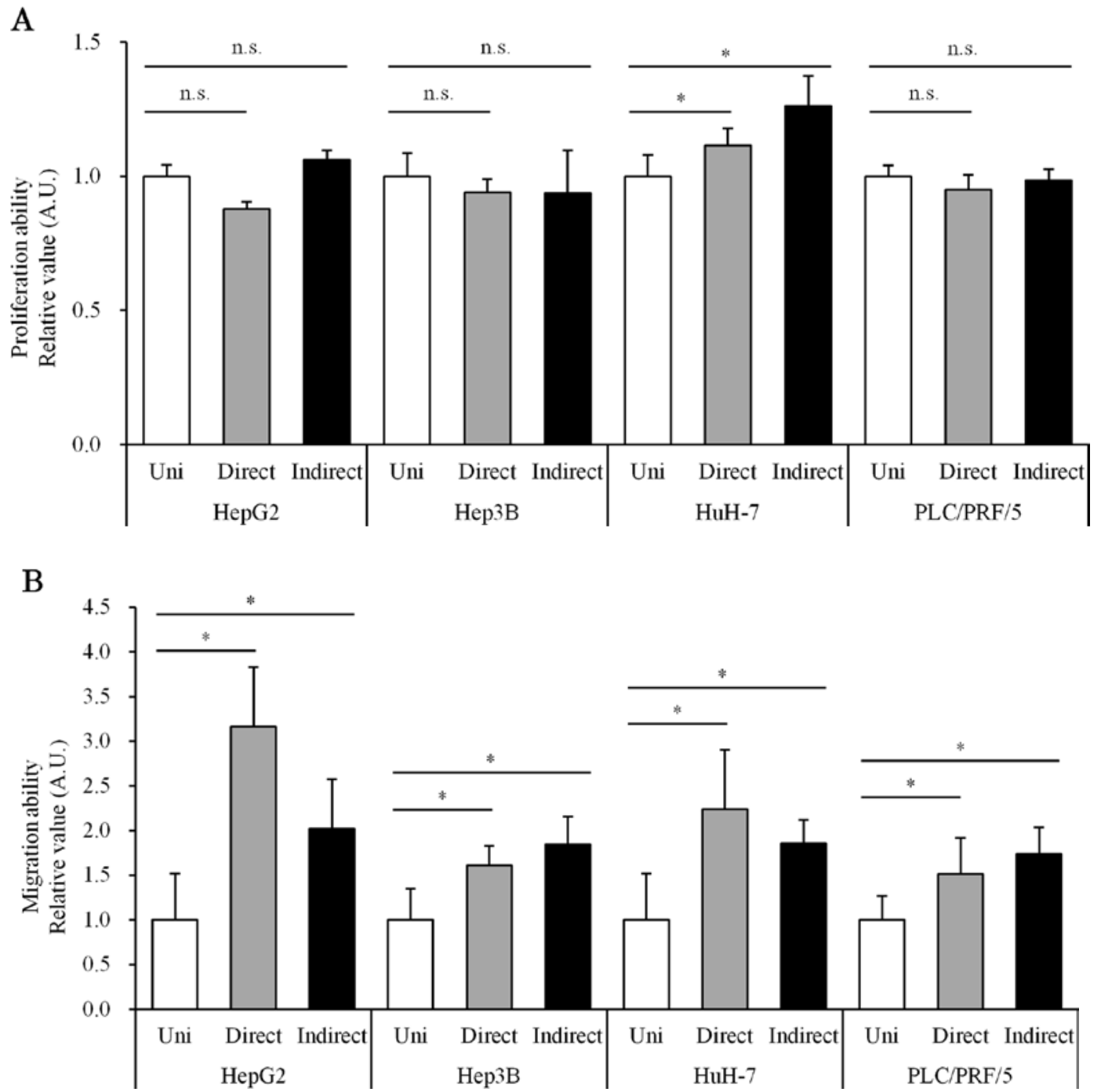

Figure 1. Biological effects in HCC cell lines under co-culture with HSCs. (A) HCC cell lines were directly and indirectly co-cultured with HSCs. Indirect co-culture was performed using a Cell Culture Insert ${ }^{\mathrm{TM}}$. Cell proliferative abilities in HCC cell lines were evaluated by flow cytometry. (B) Cell migration activity was measured as described in Materials and methods. Data represent the mean \pm SD of $>3$ independent experiments, each performed in triplicate. n.s., $\mathrm{p}>0.05$; " $\mathrm{p}<0.05$ (vs., data for uni-culture); Student's t-test. HCC, hepatocellular carcinoma; HSCs, hepatic stellate cells.

incubation was continued for an additional $48 \mathrm{~h}$ in DMEM medium supplemented with $0.5 \%$ dialyzed FBS. Images were captured at 0,24 and $48 \mathrm{~h}$, and the distance traveled by the cells at the acellular front was measured in 3 places.

Flow cytometric analysis. Cells were seeded in 6-well culture plates and uni-cultured, directly or indirectly co-cultured with HSCs in DMEM medium supplemented with $0.5 \%$ dialyzed FBS for 3 days. They were subsequently washed twice with DPBS, collected and re-suspended in $100 \mu \mathrm{l}$ of S-PBS (DPBS supplemented with $2 \%$ FBS and 10 mM HEPES buffer saline solution). Vio-blue-anti-CD44, PE-vio770anti-CD133, APC-anti-EpCAM, APC-vio770-anti-E-cadherin (130-104-270, 130-104-155, 130-091-254 and 130-101-095; Miltenyi Biotechnology, Bergisch Gladbach, Germany), FITC-anti-CD90 (IM1839U; Beckman Coulter, Brea, CA, USA), and PE-anti-N-cadherin antibodies (350806; BioLegend, San Diego, CA, USA) were added, and cells were incubated on ice for $15 \mathrm{~min}$ in the dark. After washing once, cells were re-suspended in $500 \mu \mathrm{l}$ of S-PBS supplemented with $5 \mu \mathrm{l}$ of propidium iodide (PI) solution (P378; Dojindo, Kumamoto, Japan) and analyzed using a flow cytometer (MACSQuant, Miltenyi Biotechnology).

Statistical analysis. The results are expressed as mean \pm SD. Experiments were performed at least 3 times. Differences between the groups were evaluated by the Student's t-test. A p-value $<0.05$ was considered to indicate a statistically significant result.

\section{Results}

Direct or indirect co-culture with HSCs does not increase proliferation in HCC cell lines. To assess the biological effect of co-culture with HSCs, the proliferative activities of HCC cell lines were assessed by flow cytometry. This technique allowed us to evaluate the number of viable HCC cells in the mixture. The proliferation ability of the co-culture group was not significantly different from that of the uni-culture group (Fig. 1A). 


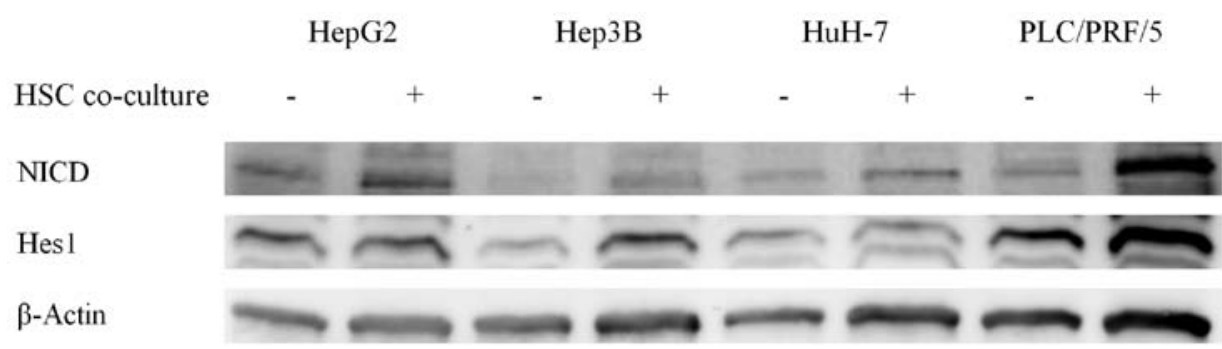

Figure 2. The effect of HSC co-culture on Notch signaling in HCC cell lines. HCC cell lines were co-cultured with HSC using a cell culture insert. After removing HSC cells, lysates were prepared from HCC cells. Immunoblot analyses were performed using antibodies against NICD and Hes1. Immunoblotting for $\beta$-actin levels was used to verify equal loading of cellular proteins. Representative blots of $>3$ independent experiments are shown. HSC, hepatic stellate cell; HCC, hepatocellular carcinoma.

Direct and indirect co-culture with HSCs promotes migration in HCC cell lines. We evaluated the migration ability of the uni- and co-culture groups using an in vitro wound healing assay. The migration activity under co-culture conditions was higher than that under uni-culture condition in all four HCC cell lines (Fig. 1B). This effect was observed in both direct and indirect co-culture conditions (Fig. 1B).

Indirect co-culture with HSCs upregulates Notch signaling in HCC cell lines. As cell migration ability was increased by co-culture with HSCs, we hypothesized that the co-culture condition enhanced EMT in HCC cell lines. The Notch signaling pathway exists upstream of EMT and regulates it. Therefore, we evaluated the expression of proteins in the Notch signaling pathway by immunoblotting. Notch intracellular domain (NICD) and Hes1 expression were increased in the co-culture group, indicating activation of the Notch signaling pathway (Fig. 2).

Indirect co-culture with HSCs promotes EMT in HCC cell lines. It is known that the expression of EpCAM and Twist are increased in EMT $(17,18)$. We evaluated the expression of the EMT markers Twist1, E-cadherin, N-cadherin and EpCAM by immunoblotting in the uni- and co-culture groups. Both EpCAM and Twist1 expression were increased in the co-culture group (Fig. 3A).

Direct and indirect co-culture with HSCs increases the proportion of EpCAM-positive HCC cells. We evaluated the expression of E-cadherin and N-cadherin, both of which are associated with EMT, by immunoblotting in the uni- and co-culture groups. The co-culture reduced E-cadherin expression in HepG2 and Hep3B, and induced N-cadherin expression in HepG2, Hep3B, HuH-7 and PLC/PRF/5. As we suspected that only a portion of the cells changed their EMT-marker expression, we evaluated the expressions of EpCAM, E-cadherin and N-cadherin by flow cytometry in both groups. In the co-culture group, we found that the ratio of EpCAM-positive cells increased (Fig. 3B). Furthermore, the percentage of low E-cadherin and high $\mathrm{N}$-cadherin cells increased, suggesting the progression of EMT in the co-culture group (Fig. 3C).

Suppression of TGF- $\beta$ and HB-EGF inhibits migration, Notch signaling. Enhanced migration activity and EMT progression were observed in both direct and indirect co-culture groups, changes that we suspected were caused by liquid factors. With the aim of reducing the liquid factors secreted from HSCs, such as TGF- $\beta$ and HB-EGF, we treated HSCs with siRNA against these two molecules.

The siRNA against TGF- $\beta$ and HB-EGF successfully suppressed TGF- $\beta(51.7 \pm 20.2 \%$ inhibition) and HB-EGF $(69.2 \pm 12.3 \%$ inhibition) expression in HSC cell lines, respectively. Both TGF- $\beta$ and HB-EGF siRNA had a minimal effect on cell proliferative activity in HCC cell lines co-cultured with HSCs (Fig. 4A). However, both TGF- $\beta$ and HB-EGF siRNA reduced the cell migration potential of HCC cell lines under co-culture with HSCs (Fig. 4B).

The expression of proteins in the Notch signaling pathway was analyzed by immunoblotting. Migration activity, and expression of proteins associated with the Notch signaling pathway were decreased in HCC cells treated with siRNA against TGF- $\beta$ and HB-EGF compared to the control (Fig. 4C).

Suppression of TGF- $\beta$ and $H B-E G F$ reduces EpCAM-positive $H C C$ cells and inhibits EMT. We performed flow cytometry to evaluate EMT capability and stemness of HCC cell lines co-cultured with HSCs treated with siRNA against TGF- $\beta$ and HB-EGF. Both TGF- $\beta$ and HB-EGF siRNA decreased the population of EpCAM-positive HCC cells (Fig. 5A). The population of HCC cells expressing $\mathrm{N}$-cadherin but lacking E-cadherin occurring in EMT was decreased (Fig. 5B).

\section{Discussion}

Cancerous tumors consist of heterogeneous cancer and stromal cells. The stromal cell-associated cancer microenvironment is known to play critical roles in cancer growth and progression $(11,19)$. In the present study, we demonstrated that HSCs induce EMT in HCC cells. HSCs were chosen among various hepatic stromal cells as they secrete various growth factors and directly affect hepatocytes (20). The immunohistochemical analyses revealed that HSCs were present in the stroma of HCC (16). Furthermore, the activation of HSCs in the medium promoted tumorigenicity of HCC (21). It is also reported that activated peritumoral HSCs are connected to high and early tumor recurrence, and increased death rate (22). Collectively, the evidence suggests that HSCs contribute to HCC progression.

We first assessed the biological impact on HCC cells under co-culture conditions with HSC (Fig. 1). We observed 
A

HepG2

Hep3B

HuH-7

PLC/PRF/5

HSC co-culture

Twist 1

EpCAM

E-Cadherin

N-Cadherin

$\beta$-Actin

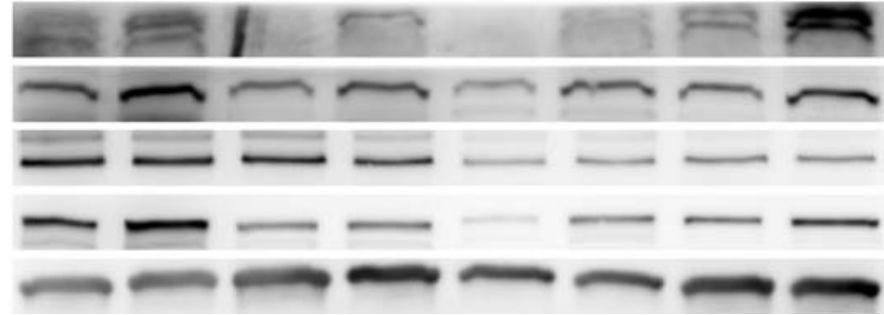

B Hep3B
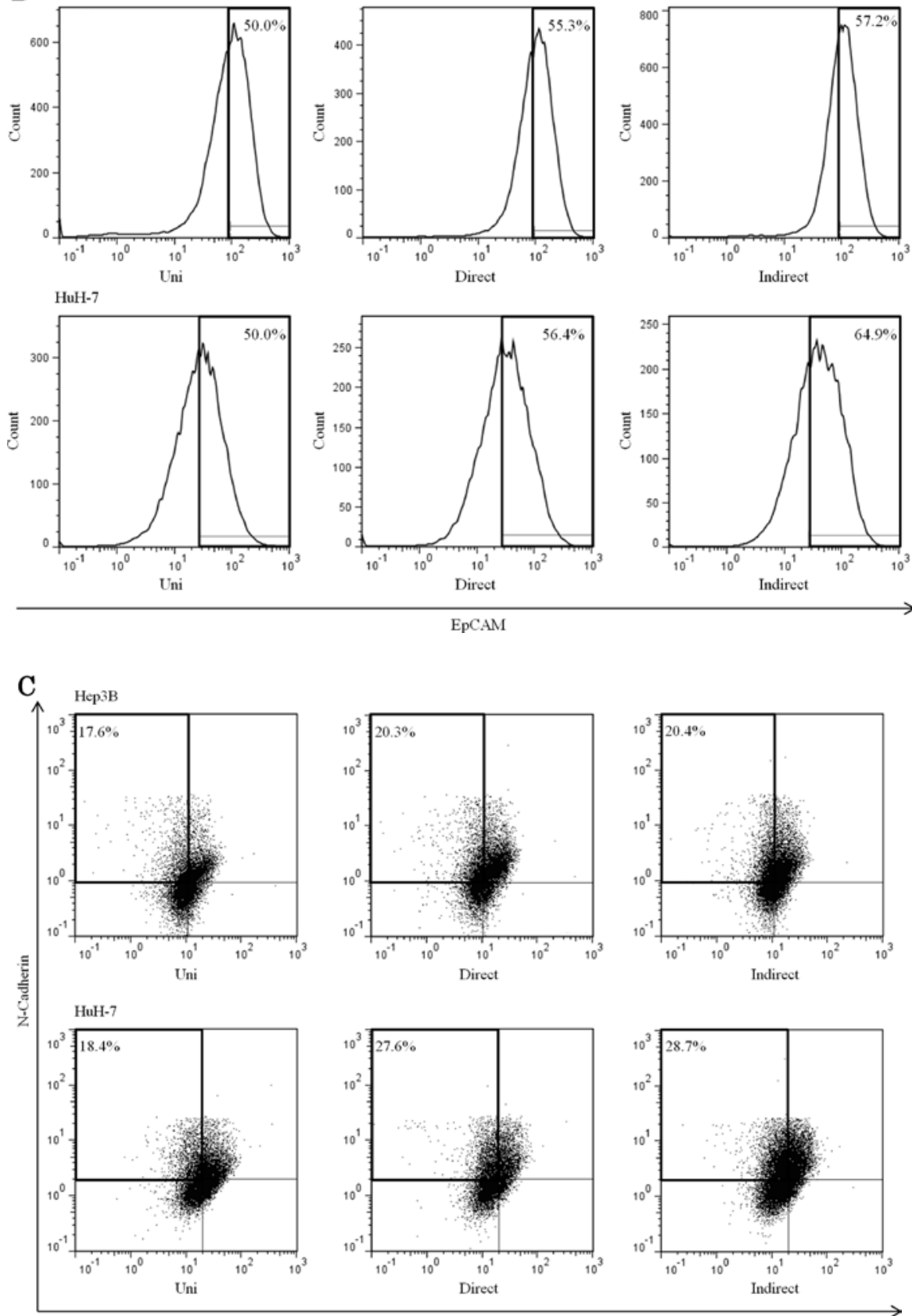

F-Cadherin

Figure 3. The effect of HSC co-culture on EMT in HCC cell lines. HCC cell lines were co-cultured with HSC using a Cell Culture Insert ${ }^{\mathrm{TM}}$. (A) After $72 \mathrm{~h}$ co-culture, HCC cells were lysed and used for immunoblot analysis. Immunoblot analyses were performed using antibodies against Twist1, E-cadherin, $\mathrm{N}$-cadherin and EpCAM. Immunoblotting for $\beta$-actin was used to verify equal loading of cellular proteins. Representative blots of $>3$ independent experiments are shown. (B) HCC cells were collected and washed in PBS without $\mathrm{Ca}^{2+} / \mathrm{Mg}^{2+}$ and incubated with APC-conjugated anti-EpCAM antibody. The cells were analyzed with MACSQuant flow cytometer. The representative plots from $>3$ independent experiments are shown. (C) HCC cells were collected and washed in PBS without $\mathrm{Ca}^{2+} / \mathrm{Mg}^{2+}$ and incubated with APC-vio770-conjugated anti-E-cadherin and PE-conjugated anti-N-cadherin. The cells were analyzed with the flow cytometer. The representative plots from $>3$ independent experiments are shown. HSC, hepatic stellate cell; EMT, epithelial-mesenchymal transition; HCC, hepatocellular carcinoma; EpCAM, epithelial cell adhesion molecule. 


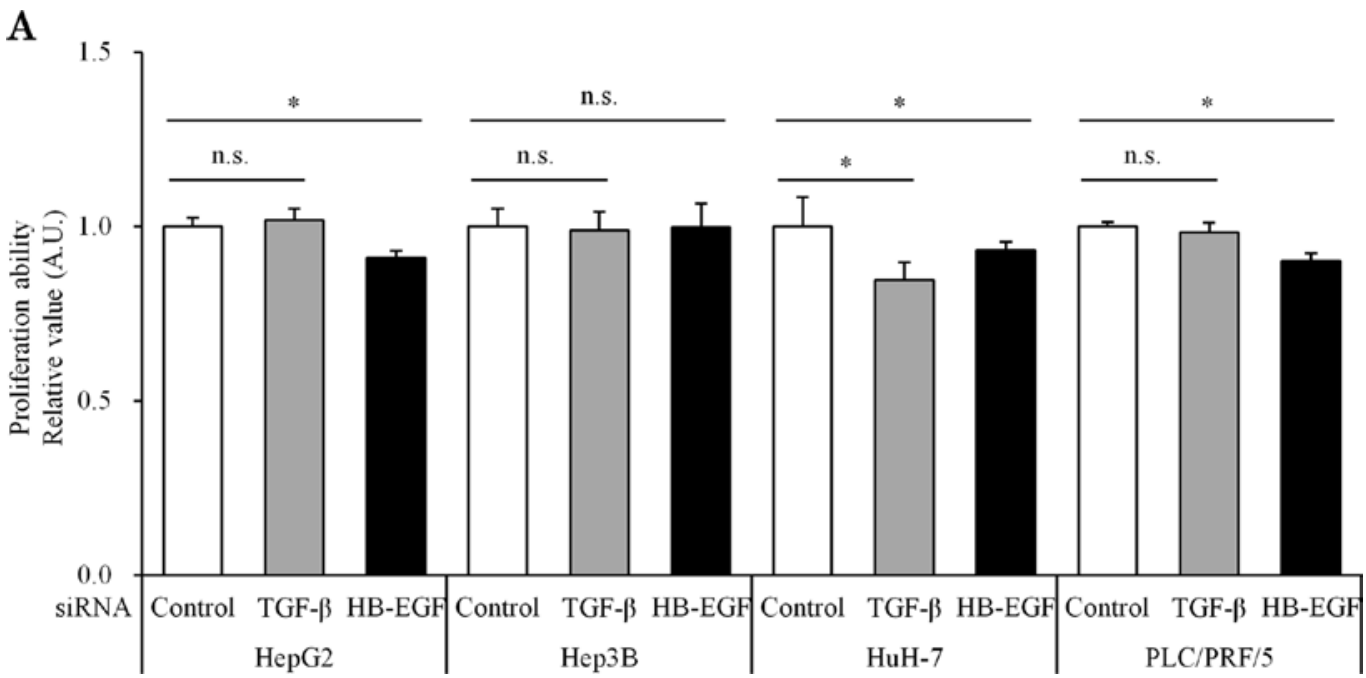

B

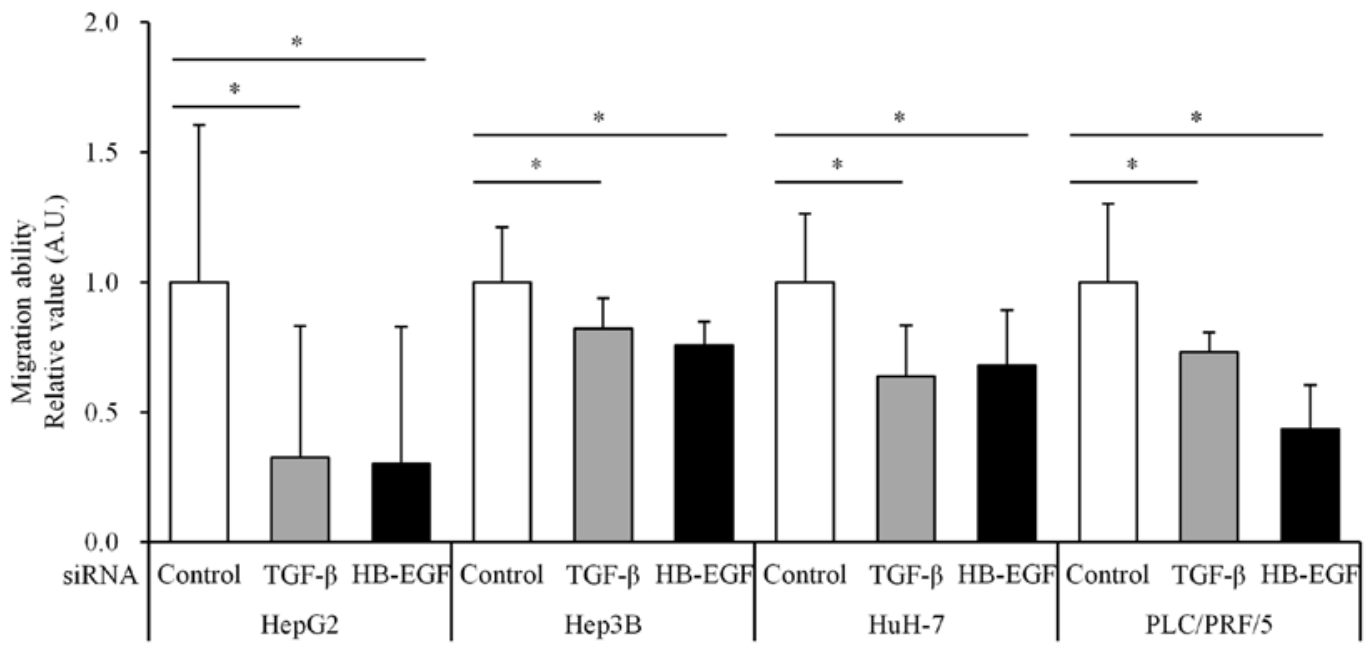

C

HepG2 Hep3B HuH-7 $\quad$ PLC/PRF/5

siRNA Control TGF- $\beta$ HB-EGF Control TGF- $\beta$ HB-EGF Control TGF- $\beta$ HB-EGF Control TGF- $\beta$ HB-EGF

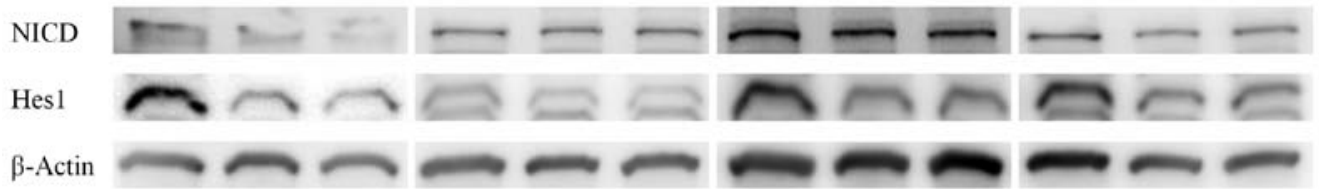

Figure 4. Effect of TGF- $\beta$ and HB-EGF gene silencing on HCC cell lines co-cultured with HSCs. HSCs were treated with TGF- $\beta 1 / 2 / 3$ and HB-EGF siRNA for $5 \mathrm{~h}$. Then, HCC cell lines were co-cultured with siRNA-treated HSCs for $48 \mathrm{~h}$. (A) Cell proliferation activity was evaluated by MTT assay. Data represent the mean $\pm \mathrm{SD}$ of $>3$ independent experiments, each performed in triplicate. n.s., $\mathrm{p}>0.05$; " $\mathrm{p}<0.05$ (vs. data for control siRNA treated group); Student's t-test. (B) Cell migration activity was assessed by an in vitro wound healing assay. Data represent the mean \pm SD of $>3$ independent experiments, each performed in triplicate. n.s., p $>0.05 ;{ }^{*} \mathrm{p}<0.05$ (vs. data for control siRNA treated group); Student's t-test. (C) HCC cells were isolated and lysed. Immunoblot analysis was performed using antibodies against NICD and Hes1. Immunoblotting for $\beta$-actin was used to verify equal loading of cellular proteins. Representative blots of $>3$ independent experiments are shown. HCC, hepatocellular carcinoma; HSCs, hepatic stellate cells.

that the co-culture did not affect the proliferation of HCC cells (Fig. 1A), while the migration ability of HCC cells was significantly enhanced (Fig. 1B). This effect was observed in both direct and indirect co-culture experiments, suggesting that this effect was induced by soluble factors.

It is reported that glioblastoma and cholangiocarcinoma cells with their respective astrocytes enhance EMT and stemness in cancer cells $(13,14)$. Pancreatic cancer cell lines are also reported to enhance EMT when cultured with pancreatic stellate cells (15). The growing body of evidence indicates that the stromal microenvironment contributes to cancer progression. In accordance with these studies, the HSC-related microenvironment induced EMT in HCC cells in the present study.

EMT plays an important role in cancer progression as well as normal embryogenesis (23). The cancer microenvironment 
A
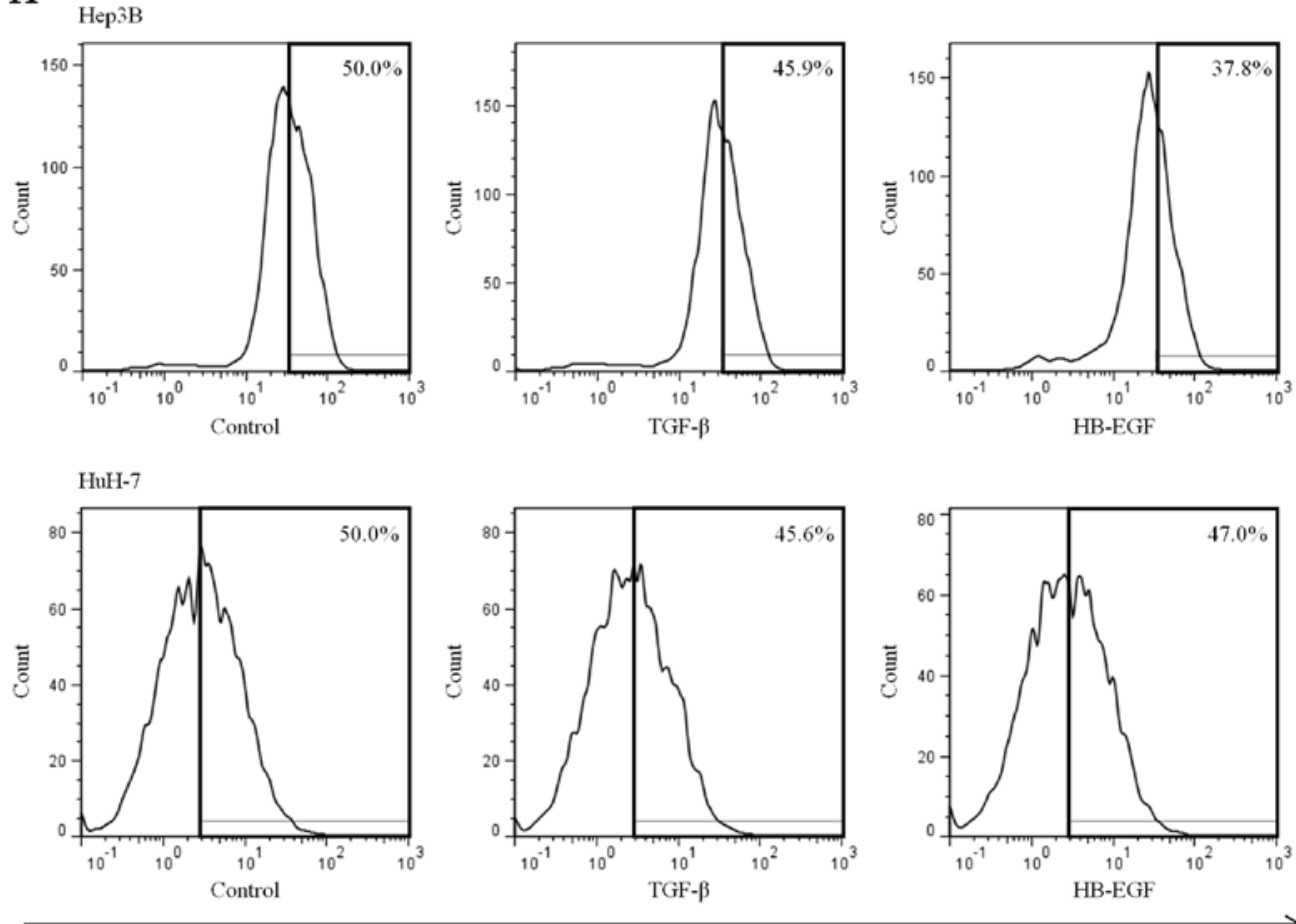

EpCAM

\section{B}

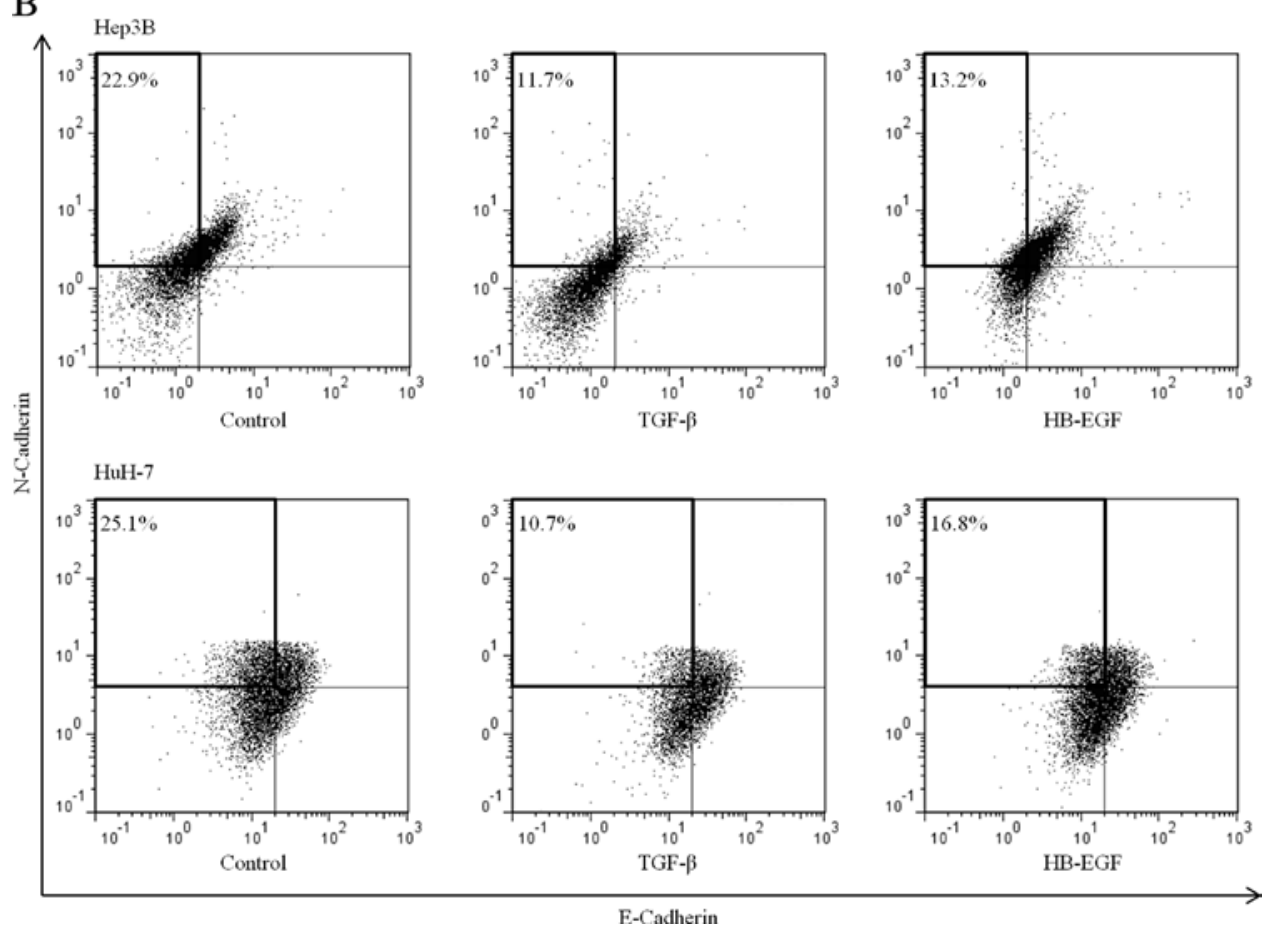

Figure 5. Effect on EMT and stemness of TGF- $\beta$ and HB-EGF gene silencing in HCC cell lines co-cultured with HSCs. (A) HSCs were treated with TGF- $\beta 1 / 2 / 3$ and HB-EGF siRNA for $5 \mathrm{~h}$. Then, HCC cell lines were co-cultured with siRNA-treated HSCs for $72 \mathrm{~h}$. HCC cells were collected and washed in PBS without $\mathrm{Ca}^{2+} / \mathrm{Mg}^{2+}$ and incubated with APC-conjugated anti-EpCAM antibody. The cells were analyzed with MACSQuant flow cytometer. Representative plots from $>3$ independent experiments are shown. (B) HSCs were treated with TGF- $\beta 1 / 2 / 3$ and HB-EGF siRNA for 5 h. Then, HCC cell lines were co-cultured with siRNA-treated HSCs for $72 \mathrm{~h}$. HCC cells were collected and washed in PBS without $\mathrm{Ca}^{2+} / \mathrm{Mg}^{2+}$ and incubated with APC-vio770-conjugated anti-E-cadherin and PE-conjugated anti-N-cadherin. The cells were analyzed with the flow cytometer. Representative plots from $>3$ independent experiments are shown. EMT, epithelial-mesenchymal transition; HCC, hepatocellular carcinoma; HSCs, hepatic stellate cells; EpCAM, epithelial cell adhesion molecule.

contributes to cancer progression and induces the occurrence of EMT in cancer cells $(11,24)$. As the migration ability is closely associated with EMT, we conducted an immunoblot analysis for EMT-related molecules (Figs. 2 and 3A). The co-culture condition decreased the expression of E-cadherin in HCC cells and increased the expression of $\mathrm{N}$-cadherin. Notably, the 
co-culture condition also induced the expression of Twist, a strong EMT inducer and EpCAM in HCC cells (Fig. 3A).

Our previous study demonstrated that the expression of EpCAM is suppressed by the expression of runt-related transcription factor 3 (RUNX3) and that the Notch signaling pathway is reduced by RUNX3 expression (8). This evidence suggests that EpCAM expression and Notch signaling is closely related. We subsequently assessed the effect of co-culture on Notch signaling (Fig. 2). Co-culture conditions activated a downstream signaling molecule of Notch in HCC cells, as expected (Fig. 2).

In order to analyze the population of EMT-induced cells, flow cytometric analysis was conducted, which revealed that the co-culture increased the ratio of EpCAM-positive cells in Hep3B and HuH-7 (Fig. 3B). The population of cells with low expression of E-cadherin and high expression of $\mathrm{N}$-cadherin was increased in co-culture, compared to the uni-culture condition (Fig. 3C). These results indicated that the co-culture with HSC activated the Notch signaling pathway and induced EMT in HCC cells. In these experiments, both direct and indirect co-culture condition demonstrated similar results, indicating that the Notch signal activation and EMT were induced by soluble factors.

A number of soluble mitogens are secreted by HSCs (20). As TGF- $\beta$ and HB-EGF are strong EMT inducers, we performed gene silencing experiments using siRNAs against them. These siRNA-treated HSCs were cultured with HCC, and their proliferation and migration abilities were assessed (Fig. 4). The siRNAs successfully silenced the expression of TGF- $\beta$ and HB-EGF, resulting in decreased cell migration ability in the co-culture (Fig. 4B).

Flow cytometric analysis confirmed that the siRNA also reduced EMT in HCC cells under the co-culture with HSCs (Fig. 5). The results indicate that the interactions between HSCs and HCC cells were mainly induced by the soluble factors secreted by HSCs.

The effect of HSC co-culture was relatively weak in the immunoblot analysis, presumably as a result of the low concentration of the soluble factors. The Cell Culture Insert $^{\mathrm{TM}}$ allowed us to assess the effect of soluble factors in the co-culture system while excluding the effect of cell-cell contact. The limitation of this co-culture system, however, is the relatively low concentration of soluble factors. The co-culture system required at least $3 \mathrm{ml}$ of medium, which generates a low concentration of the soluble factors produced by $5 \times 10^{4}$ HSC cells.

Another limitation of the present study is the use of immortalized HSCs. HSC cell line TWNT-1 may behave differently from natural HSCs, although TWNT-1 is known to have features similar to those exhibited by activated HSCs (25). This may be an advantage for elucidating the mechanism of HCC progression, since the active form of HSC contributes to it $(21,26)$. Further studies are necessary to elucidate the role of HSC in vivo.

In advanced and metastatic $\mathrm{HCC}$, the conventional treatment modality cannot achieve a curative effect. EMT and cancer stemness contributed to the poor prognosis in $\mathrm{HCC}(3-5,12,27)$. The activation of Notch signaling contributes to both EMT and stemness. Our results showed that HSCs activate the Notch signaling pathway and induce EMT, which indicates that HSCs may also activate CSCs. However, EpCAM-positive HCC cells were shown to have stem cell properties such as self-renewal, differentiation and tumor-initiating ability (6). It was also reported that higher EpCAM expression was related to the development of chemoresistance in HCC (28). Taken together, therefore, EpCAM has become recognized as a CSC marker in HCC $(6,28)$. The interaction between HSCs and $\mathrm{HCC}$ cells may induce cancer progression by activating EMT and stemness. CSCs are resistant to the conventional therapeutic modality for HCC (29). A number of studies have demonstrated that high expression levels of CSC markers are related to the poor prognosis of HCC (3-5). Others have uncovered that EpCAM expression is also associated with poor prognosis (3-5). Thus, the interactions between HSC and HCC cells could be a therapeutic target for HCC. Further studies are necessary, however, to elucidate the effects induced by HSCs, particularly on CSCs.

In conclusion, we evaluated the effect of the cancer microenvironment on $\mathrm{HCC}$ by in vitro co-culture assay with HSCs. TGF- $\beta$ and HB-EGF secreted by HSCs were shown to promote the acquirement of EMT and a stem cell phenotype by HCCs. TGF- $\beta$ and HB-EGF could therefore be promising new therapeutic targets for CSC-based therapy.

\section{Acknowledgements}

The authors wish to thank Dr Naoya Kobayashi for his generous gift of immortalized human HSC cells (TWNT-1).

\section{References}

1. European Association For The Study Of The Liver; European Organisation For Research And Treatment Of Cancer: EASL-EORTC clinical practice guidelines: Management of hepatocellular carcinoma. J Hepatol 56: 908-943, 2012.

2. Venook AP, Papandreou C, Furuse J and de Guevara LL: The incidence and epidemiology of hepatocellular carcinoma: A global and regional perspective. Oncologist 15 (Suppl 4): S5-S13, 2010.

3. Bae JS, Noh SJ, Jang KY, Park HS, Chung MJ, Park CK and Moon WS: Expression and role of epithelial cell adhesion molecule in dysplastic nodule and hepatocellular carcinoma. Int J Oncol 41: 2150-2158, 2012.

4. Schulze K, Gasch C, Staufer K, Nashan B, Lohse AW, Pantel K, Riethdorf S and Wege H: Presence of EpCAM-positive circulating tumor cells as biomarker for systemic disease strongly correlates to survival in patients with hepatocellular carcinoma. Int J Cancer 133: 2165-2171, 2013.

5. Chan AW, Tong JH, Chan SL, Lai PB and To KF: Expression of stemness markers (CD133 and EpCAM) in prognostication of hepatocellular carcinoma. Histopathology 64: 935-950, 2014.

6. Yamashita T, Ji J, Budhu A, Forgues M, Yang W, Wang HY, Jia H, Ye Q, Qin LX, Wauthier E, et al: EpCAM-positive hepatocellular carcinoma cells are tumor-initiating cells with stem/progenitor cell features. Gastroenterology 136: 1012-1024, 2009.

7. Bao B, Wang Z, Ali S, Kong D, Li Y, Ahmad A, Banerjee S, Azmi AS, Miele L and Sarkar FH: Notch-1 induces epithelial-mesenchymal transition consistent with cancer stem cell phenotype in pancreatic cancer cells. Cancer Lett 307: 26-36, 2011.

8. Nishina S, Shiraha H, Nakanishi Y, Tanaka S, Matsubara M, Takaoka N, Uemura M, Horiguchi S, Kataoka J, Iwamuro M, et al: Restored expression of the tumor suppressor gene RUNX3 reduces cancer stem cells in hepatocellular carcinoma by suppressing Jagged1-Notch signaling. Oncol Rep 26: 523-531, 2011.

9. van der Gun BT, Melchers LJ, Ruiters MH, de Leij LF, McLaughlin PM and Rots MG: EpCAM in carcinogenesis: The good, the bad or the ugly. Carcinogenesis 31: 1913-1921, 2010. 
10. Patriarca C, Macchi RM, Marschner AK and Mellstedt $\mathrm{H}$ : Epithelial cell adhesion molecule expression (CD326) in cancer: A short review. Cancer Treat Rev 38: 68-75, 2012.

11. Wu SD, Ma YS, Fang Y, Liu LL, Fu D and Shen XZ: Role of the microenvironment in hepatocellular carcinoma development and progression. Cancer Treat Rev 38: 218-225, 2012.

12. Brabletz T: To differentiate or not - routes towards metastasis Nat Rev Cancer 12: 425-436, 2012.

13. Okamoto K, Tajima H, Nakanuma S, Sakai S, Makino I, Kinoshita J, Hayashi H, Nakamura K, Oyama K, Nakagawara H, et al: Angiotensin II enhances epithelial-to-mesenchymal transition through the interaction between activated hepatic stellate cells and the stromal cell-derived factor-1/CXCR4 axis in intrahepatic cholangiocarcinoma. Int J Oncol 41: 573-582, 2012.

14. Kim SW, Choi HJ, Lee HJ, He J, Wu Q, Langley RR, Fidler IJ and Kim SJ: Role of the endothelin axis in astrocyte- and endothelial cell-mediated chemoprotection of cancer cells. Neuro Oncol 16: $1585-1598,2014$.

15. Kikuta K, Masamune A, Watanabe T, Ariga H, Itoh H, Hamada S, Satoh K, Egawa S, Unno M and Shimosegawa T: Pancreatic stellate cells promote epithelial-mesenchymal transition in pancreatic cancer cells. Biochem Biophys Res Commun 403: 380-384, 2010.

16. Hellerbrand C: Hepatic stellate cells - the pericytes in the liver. Pflugers Arch 465: 775-778, 2013

17. Osta WA, Chen Y, Mikhitarian K, Mitas M, Salem M, Hannun YA, Cole DJ and Gillanders WE: EpCAM is overexpressed in breast cancer and is a potential target for breast cancer gene therapy. Cancer Res 64: 5818-5824, 2004.

18. Yang J, Mani SA, Donaher JL, Ramaswamy S, Itzykson RA, Come C, Savagner P, Gitelman I, Richardson A and Weinberg RA Twist, a master regulator of morphogenesis, plays an essential role in tumor metastasis. Cell 117: 927-939, 2004.

19. Coulouarn C, Corlu A, Glaise D, Guénon I, Thorgeirsson SS and Clément B: Hepatocyte-stellate cell cross-talk in the liver engenders a permissive inflammatory microenvironment that drives progression in hepatocellular carcinoma. Cancer Res 72 2533-2542, 2012.
20. Friedman SL: Hepatic stellate cells: Protean, multifunctional, and enigmatic cells of the liver. Physiol Rev 88: 125-172, 2008.

21. Amann T, Bataille F, Spruss T, Mühlbauer M, Gäbele E, Schölmerich J, Kiefer P, Bosserhoff AK and Hellerbrand C: Activated hepatic stellate cells promote tumorigenicity of hepatocellular carcinoma. Cancer Sci 100: 646-653, 2009.

22. Ju MJ, Qiu SJ, Fan J, Xiao YS, Gao Q, Zhou J, Li YW and Tang ZY: Peritumoral activated hepatic stellate cells predict poor clinical outcome in hepatocellular carcinoma after curative resection. Am J Clin Pathol 131: 498-510, 2009.

23. Samatov TR, Tonevitsky AG and Schumacher U: Epithelialmesenchymal transition: Focus on metastatic cascade, alternative splicing, non-coding RNAs and modulating compounds. Mol Cancer 12: 107, 2013.

24. Tse JC and Kalluri R: Mechanisms of metastasis: Epithelialto-mesenchymal transition and contribution of tumor microenvironment. J Cell Biochem 101: 816-829, 2007.

25. Watanabe T, Shibata N, Westerman KA, Okitsu T, Allain JE, Sakaguchi M, Totsugawa T, Maruyama M, Matsumura T, Noguchi H, et al: Establishment of immortalized human hepatic stellate scavenger cells to develop bioartificial livers. Transplantation 75: 1873-1880, 2003.

26. Kang N, Gores GJ and Shah VH: Hepatic stellate cells: Partners in crime for liver metastases? Hepatology 54: 707-713, 2011.

27. Hashiguchi M, Ueno S, Sakoda M, Iino S, Hiwatashi K, Minami K, Ando K, Mataki Y, Maemura K, Shinchi H, et al: Clinical implication of ZEB-1 and E-cadherin expression in hepatocellular carcinoma (HCC). BMC Cancer 13: 572, 2013

28. Kimura O, Kondo Y, Kogure T, Kakazu E, Ninomiya M, Iwata T, Morosawa $\mathrm{T}$ and Shimosegawa T: Expression of EpCAM increases in the hepatitis B related and the treatment-resistant hepatocellular carcinoma. BioMed Res Int 2014: 172913, 2014.

29. Oishi N, Yamashita T and Kaneko S: Molecular biology of liver cancer stem cells. Liver Cancer 3: 71-84, 2014. 